\title{
ARENS REGULARITY OF MODULE ACTIONS AND THE SECOND ADJOINT OF A DERIVATION
}

\author{
S. MOHAMMADZADEH and H. R. E. VISHKI ${ }^{\otimes}$
}

(Received 15 August 2007)

\begin{abstract}
In this paper, we give a simple criterion for the Arens regularity of a bilinear mapping on normed spaces, which applies in particular to Banach module actions, and then investigate those conditions under which the second adjoint of a derivation into a dual Banach module is again a derivation. As a consequence of the main result, a simple and direct proof for several older results is also included.
\end{abstract}

2000 Mathematics subject classification: 46H20, 46H25.

Keywords and phrases: Arens product, bounded bilinear map, Banach module action, derivation, module action, second dual.

\section{Introduction}

In his pioneering paper [3], Arens showed that a bounded bilinear map $f: X \times Y \longrightarrow$ $Z$ on normed spaces has two natural but, in general, different extensions to the bilinear maps $f^{* * *}$ and $f^{r * * * r}$. When these extensions are equal, $f$ is said to be (Arens) regular. If the multiplication of a Banach algebra $A$ enjoys this property, then $A$ itself is called (Arens) regular.

In this paper we first provide a criterion for the regularity of a bounded bilinear map (Theorem 2.1 below), by showing that $f$ is regular if and only if $f^{* * * *}\left(Z^{*}, X^{* *}\right) \subseteq Y^{*}$, which in turn covers some older results of $[5,10]$ on this topic (see Corollaries 2.2 and 2.3 below). Then we apply the above-mentioned criterion for the module actions of a Banach $A$-module $X$, which in turn give rise to the module actions of $A^{* *}$ (equipped with each of the Arens products) on $X^{* *}$ and $X^{* * *}$, in a natural way. In this direction we present Propositions 3.1, 3.3 and 3.6, which generalize some results of $[4,6,10,11]$.

For a Banach $A$-module $X$, the second adjoint $D^{* *}: A^{* *} \rightarrow X^{* * *}$ of a derivation $D: A \rightarrow X^{*}$ is trivially a linear extension of $D$. A problem which is of interest is under what conditions $D^{* *}$ is again a derivation. Dales et al. in [10] studied this problem for the special case $X=A$, and they showed that $D^{* *}$ is a derivation if and only if

(c) 2008 Australian Mathematical Society 0004-9727/08 \$A2.00+0.00 
$D^{* *}\left(A^{* *}\right) \cdot A^{* *} \subseteq A^{*}$ (see [10, Theorem 7.1]). We extend their result for a general derivation $D: A \rightarrow X^{*}$ with a direct proof (see Theorem 4.2 below). This theorem in turn extends some other results of $[6,10]$ for a general derivation $D: A \rightarrow X^{*}$.

For terminology and background materials we follow [8] as far as possible.

\section{Arens regularity of bilinear maps}

For a normed space $X$, we denote by $X^{*}$ the topological dual of $X$. We write $X^{* *}$ for $\left(X^{*}\right)^{*}$, and so on. Throughout the paper, we usually identify a normed space with its canonical image in its second dual.

Let $X, Y$ and $Z$ be normed spaces and let $f: X \times Y \longrightarrow Z$ be a bounded bilinear map. The adjoint $f^{*}: Z^{*} \times X \longrightarrow Y^{*}$ of $f$ is defined by

$$
\left\langle f^{*}\left(z^{*}, x\right), y\right\rangle=\left\langle z^{*}, f(x, y)\right\rangle \quad\left(x \in X, y \in Y, z^{*} \in Z^{*}\right),
$$

which is also a bounded bilinear map. By setting $f^{* *}=\left(f^{*}\right)^{*}$ and continuing in this way, the mappings $f^{* *}: Y^{* *} \times Z^{*} \longrightarrow X^{*}, f^{* * *}: X^{* *} \times Y^{* *} \longrightarrow Z^{* *}$ and $f^{* * * *}: Z^{* * *} \times X^{* *} \longrightarrow Y^{* * *}$ may be defined similarly.

The mapping $f^{* * *}$ is the unique extension of $f$ such that $f^{* * *}\left(\cdot, y^{* *}\right)$ is $w^{*}$ continuous for every $y^{* *} \in Y^{* *}$. Also $f^{* * *}(x, \cdot)$ is $w^{*}$-continuous for every $x \in X$.

We also denote by $f^{r}$ the flip map of $f$, that is, the bounded bilinear map $f^{r}: Y \times X \longrightarrow Z$ defined by $f^{r}(y, x)=f(x, y)(x \in X, y \in Y)$. It may be extended as above to $f^{r * * * r}: X^{* *} \times Y^{* *} \longrightarrow Z^{* *}$ which in turn is a unique extension of $f$ such that $f^{r * * * r}\left(x^{* *}, \cdot\right)$ is $w^{*}$-continuous for every $x^{* *} \in X^{* *}$, and also $f^{r * * * r}(\cdot, y)$ is $w^{*}$ continuous for every $y \in Y$. One may also easily verify that

$$
f^{* * *}\left(x^{* *}, y^{* *}\right)=w^{*}-\lim _{\alpha} \lim _{\beta} f\left(x_{\alpha}, y_{\beta}\right)
$$

and

$$
f^{r * * * r}\left(x^{* *}, y^{* *}\right)=w^{*}-\lim _{\beta} \lim _{\alpha} f\left(x_{\alpha}, y_{\beta}\right),
$$

where $\left\{x_{\alpha}\right\}$ and $\left\{y_{\beta}\right\}$ are nets in $X$ and $Y$ which converge to $x^{* *}$ and $y^{* *}$ in the $w^{*}$ topologies, respectively.

The map $f$ is called Arens regular when the equality $f^{* * *}=f^{r * * * r}$ holds.

It is easy to verify that, for the multiplication map $\pi: A \times A \longrightarrow A$ of a Banach algebra $A, \pi^{* * *}$ and $\pi^{r * * * r}$ are actually the so-called first and second Arens products [3], which will be denoted by $\square$ and $\diamond$, respectively. The Banach algebra $A$ is said to be Arens regular if the multiplication map $\pi$ is Arens regular, or equivalently $\square=\diamond$ on the whole of $A^{* *}$. This is the case, for example, for all $C^{*}$-algebras, [7], and also for $L^{1}(G)$ if (and only if) $G$ is finite, [12]. The interested reader may refer to [9] for ample information about the Arens regularity problem on a wide variety of Banach algebras.

We commence with the main theorem of this section which provides a criterion concerning to the regularity of a bounded bilinear map. 
TheOREM 2.1. For a bounded bilinear map $f: X \times Y \longrightarrow Z$ the following statements are equivalent:

(i) $f$ is regular;

(ii) $f^{* * * *}=f^{r * * * * * r}$;

(iii) $f^{* * * *}\left(Z^{*}, X^{* *}\right) \subseteq Y^{*}$;

(iv) the linear map $x \longmapsto f^{*}\left(z^{*}, x\right): X \longrightarrow Y^{*}$ is weakly compact for every $z^{*} \in Z^{*}$.

Proof. Let $x^{* *} \in X^{* *}, y^{* *} \in Y^{* *}, z^{* * *} \in Z^{* * *}$ and $z^{*} \in Z^{*}$ be arbitrary. If (i) holds then

$$
\begin{aligned}
\left\langle f^{* * * *}\left(z^{* * *}, x^{* *}\right), y^{* *}\right\rangle & =\left\langle z^{* * *}, f^{* * *}\left(x^{* *}, y^{* *}\right)\right\rangle \\
& =\left\langle z^{* * *}, f^{r * * * r}\left(x^{* *}, y^{* *}\right)\right\rangle \\
& =\left\langle z^{* * *}, f^{r * * *}\left(y^{* *}, x^{* *}\right)\right\rangle \\
& =\left\langle x^{* *}, f^{r * * * *}\left(z^{* * *}, y^{* *}\right)\right\rangle \\
& =\left\langle f^{r * * * * *}\left(x^{* *}, z^{* * *}\right), y^{* *}\right\rangle .
\end{aligned}
$$

Therefore $f^{* * * *}=f^{r * * * * * r}$, as claimed.

The implication (ii) $\Rightarrow$ (iii) follows trivially from the fact that

$$
f^{* * * *}\left(z^{*}, x^{* *}\right)=f^{r * * * * *}\left(x^{* *}, z^{*}\right)=\left.f^{r * * * * *}\right|_{X^{* *} \times Z^{*}}\left(x^{* *}, z^{*}\right)=f^{r * *}\left(x^{* *}, z^{*}\right) \in Y^{*} .
$$

That (iii) is equivalent to (iv) is obvious; indeed, if we denote $f^{*}\left(z^{*}, \cdot\right)$ by $L$, then it is easy to show that $L^{* *}=f^{* * * *}\left(z^{*}, \cdot\right)$. Now the conclusion follows from the fact that $L$ is weakly compact if and only if $L^{* *}\left(X^{* *}\right) \subseteq Y^{*}$.

For (iii) $\Rightarrow$ (i), suppose that $f^{* * * *}\left(Z^{*}, X^{* *}\right) \subseteq Y^{*}$ and let $\left\{x_{\alpha}\right\},\left\{y_{\beta}\right\}$ be two nets in $X$ and $Y$ that converge to $x^{* *}, y^{* *}$ in the $w^{*}$-topologies, respectively; then

$$
\begin{aligned}
\left\langle f^{* * *}\left(x^{* *}, y^{* *}\right), z^{*}\right\rangle & =\left\langle f^{* * * *}\left(z^{*}, x^{* *}\right), y^{* *}\right\rangle \\
& =\lim _{\beta}\left\langle f^{* * * *}\left(z^{*}, x^{* *}\right), y_{\beta}\right\rangle \\
& =\lim _{\beta}\left\langle f^{* * *}\left(x^{* *}, y_{\beta}\right), z^{*}\right\rangle \\
& =\lim _{\beta}\left\langle x^{* *}, f^{* *}\left(y_{\beta}, z^{*}\right)\right\rangle \\
& =\lim _{\beta} \lim _{\alpha}\left\langle f^{* *}\left(y_{\beta}, z^{*}\right), x_{\alpha}\right\rangle \\
& =\lim _{\beta} \lim _{\alpha}\left\langle z^{*}, f\left(x_{\alpha}, y_{\beta}\right)\right\rangle \\
& =\left\langle f^{r * * *}\left(x^{* *}, y^{* *}\right), z^{*}\right\rangle .
\end{aligned}
$$

It follows that $f$ is regular and this completes the proof.

As the first application of Theorem 2.1, we may present the following results of [10], with a direct proof. 
Corollary 2.2 [10, Propositions 4.1 and 4.4]. For a bounded bilinear map $f: X \times Y \longrightarrow Z$, the following statements are equivalent:

(i) $f$ and $f^{r *}$ are regular;

(ii) $f^{* * * r * r}=f^{r * r * * *}$;

(iii) $f^{* * * *}\left(Z^{* * *}, X^{* *}\right) \subseteq Y^{*}$.

PROOF. The implication (i) $\Rightarrow$ (ii) follows trivially.

If (ii) holds then $f^{* * * *}=f^{r * r * *}$. Indeed, for every $x^{* *} \in X^{* *}, y^{* *} \in Y^{* *}$ and $z^{* * *} \in Z^{* * *}$,

$$
\begin{aligned}
\left\langle f^{* * * *}\left(z^{* * *}, x^{* *}\right), y^{* *}\right\rangle & =\left\langle f^{* * * r * r}\left(y^{* *}, z^{* * *}\right), x^{* *}\right\rangle \\
& =\left\langle f^{r * r * * *}\left(y^{* *}, z^{* * *}\right), x^{* *}\right\rangle \\
& =\left\langle f^{r * r * *}\left(z^{* * *}, x^{* *}\right), y^{* *}\right\rangle .
\end{aligned}
$$

As $f^{r * r * *}\left(Z^{* * *}, X^{* *}\right)$ always lies in $Y^{*}$, we have reached (iii).

For (iii) $\Rightarrow$ (i), since $f^{* * * *}\left(Z^{*}, X^{* *}\right) \subseteq f^{* * * *}\left(Z^{* * *}, X^{* *}\right) \subseteq Y^{*}$, Theorem 2.1 implies the regularity of $f$, or equivalently $f^{r * * * *}=f^{* * * * r}$, from which

$$
\left(f^{r *}\right)^{* * * *}\left(X^{* *}, Z^{* * *}\right)=f^{r * * * * *}\left(X^{* *}, Z^{* * *}\right)=f^{* * * *}\left(Z^{* * *}, X^{* *}\right) \subseteq Y^{*} .
$$

Now the regularity of $f^{r *}$ follows trivially again by Theorem 2.1.

The main result of Arikan's paper, [5, Theorem 2], is a criterion for the Arens regularity of bilinear mappings which applied it to give a sequence of results on the regularity of the algebra $l^{1}$ with pointwise multiplication, the algebra $L^{\infty}(G)$ with convolution, where $G$ is a compact group, and the trace-class algebra. We now give her result as a consequence of Corollary 2.2. It is worth pointing out that the assumption that she applied in [5, Theorem 2] actually has more by-products; indeed, as we shall see in the next corollary, both $f$ and $f^{r *}$ are regular, though she merely deduces the regularity of $f$.

Corollary 2.3 (See [5, Theorem 2]). Let $f: X \times Y \longrightarrow Z$ and $g: X \times W \longrightarrow Z$ be bounded bilinear mappings and let $h: Y \longrightarrow W$ be a bounded linear mapping such that $f(x, y)=g(x, h(y))$, for all $x \in X, y \in Y$. If $h$ is weakly compact, then both $f$ and $f^{r *}$ are regular.

PROOF. Using the equality $f(x, y)=g(x, h(y))$, a standard argument applies to show that $f^{* * * *}=h^{* * *} \circ g^{* * * *}$. The weak compactness of $h$ implies that of $h^{*}$, from which we have $h^{* * *}\left(W^{* * *}\right) \subseteq Y^{*}$. Therefore

$$
f^{* * * *}\left(Z^{* * *}, X^{* *}\right)=h^{* * *}\left(g^{* * * *}\left(Z^{* * *}, X^{* *}\right)\right) \subseteq h^{* * *}\left(W^{* * *}\right) \subseteq Y^{*}
$$

Now Corollary 2.2 implies that both $f$ and $f^{r *}$ are regular, as claimed. 


\section{Arens regularity of module actions}

Let $A$ be a Banach algebra, $X$ be a Banach space and let $\pi_{1}: A \times X \longrightarrow X$ be a bounded bilinear map. Then the pair $\left(\pi_{1}, X\right)$ is said to be a left Banach $A$-module if $\pi_{1}(a b, x)=\pi_{1}\left(a, \pi_{1}(b, x)\right)$, for every $a, b \in A, x \in X$. A right Banach $A$-module $\left(X, \pi_{2}\right)$ may be defined similarly. A triple $\left(\pi_{1}, X, \pi_{2}\right)$ is said to be a Banach $A$-module if $\left(\pi_{1}, X\right)$ and $\left(X, \pi_{2}\right)$ are left and right Banach $A$-modules, respectively, and for every $a, b \in A, x \in X$,

$$
\pi_{1}\left(a, \pi_{2}(x, b)\right)=\pi_{2}\left(\pi_{1}(a, x), b\right) .
$$

If so, then trivially $\left(\pi_{2}{ }^{r * r}, X^{*}, \pi_{1}^{*}\right)$ is the dual Banach $A$-module of $\left(\pi_{1}, X, \pi_{2}\right)$. Also $\left(\pi_{1}^{* * *}, X^{* *}, \pi_{2}^{* * *}\right)$ is a Banach $\left(A^{* *}, \square\right)$-module and $\left(\pi_{1}^{r * * * r}, X^{* *}, \pi_{2}^{r * * * r}\right)$ is a Banach $\left(A^{* *}, \diamond\right)$-module. To verify these, one may carefully check that the various associativity rules follow from the analogous rules that show that $\left(\pi_{1}, X, \pi_{2}\right)$ is a Banach $A$-module.

Now if we continue dualizing we shall reach $\left(\pi_{2}^{* * * r * r}, X^{* * *}, \pi_{1}^{* * * *}\right)$ as the dual Banach $\left(A^{* *}, \square\right)$-module of $\left(\pi_{1}^{* * *}, X^{* *}, \pi_{2}^{* * *}\right)$ and $\left(\pi_{2}^{r * * * * r}, X^{* * *}, \pi_{1}^{r * * * r *}\right)$ as the dual Banach $\left(A^{* *}, \diamond\right)$-module of $\left(\pi_{1}^{r * * * r}, X^{* *}, \pi_{2}^{r * * * r}\right)$.

From now on, for a Banach $A$-module $X$ we use the above-mentioned canonical module actions on $X^{*}, X^{* *}$ and $X^{* * *}$, unless otherwise explicitly stipulated. However, for brevity of notation, when there is no risk of confusion, we may use them without the specified module actions.

According to the inclusions

$$
\pi_{2}^{* * * r * r}\left(A^{* *}, X^{*}\right)=\pi_{2}^{* *}\left(A^{* *}, X^{*}\right) \subseteq X^{*}
$$

and

$$
\pi_{1}^{r * * * r *}\left(X^{*}, A^{* *}\right)=\pi_{1}^{r * * r}\left(X^{*}, A^{* *}\right) \subseteq X^{*},
$$

we deduce that $\left(\pi_{2}^{* *}, X^{*}\right)$ is a left $\left(A^{* *}, \square\right)$-submodule of $X^{* * *}$, while $\left(X^{*}, \pi_{1}^{r * * r}\right)$ is a right $\left(A^{* *}, \diamond\right)$-submodule of $X^{* * *}$. The next result determines when $\left(\pi_{2}^{* *}, X^{*}, \pi_{1}^{r * * r}\right)$ is actually a $\left(A^{* *}, \square\right)$-submodule (or $\left(A^{* *}, \diamond\right)$-submodule) of $X^{* * *}$.

Proposition 3.1. Let $\left(\pi_{1}, X, \pi_{2}\right)$ be a Banach A-module. Then:

(i) $X^{*}$ is a $\left(A^{* *}, \square\right)$-submodule of $X^{* * *}$ if and only if $\pi_{1}$ is regular;

(ii) $X^{*}$ is a $\left(A^{* *}, \diamond\right)$-submodule of $X^{* * *}$ if and only if $\pi_{2}$ is regular.

PROOF. We prove only (i), since (ii) uses the same argument. It is trivial that $X^{*}$ is a right $\left(A^{* *}, \square\right)$-submodule of $X^{* * *}$ if and only if $\pi_{1}^{* * * *}\left(X^{*}, A^{* *}\right) \subseteq X^{*}$ and by Theorem 2.1 this is nothing more than the regularity of $\pi_{1}$; which establishes (i).

As a rapid consequence of the latter result, we examine it for $\pi_{1}=\pi$ and $\pi_{2}=\pi^{r}$ on $X=A$ and we thus have the following result of Dales et al. [10]. 
Corollary 3.2 [10, Proposition 5.2]. For a Banach algebra $A$ the following statements are equivalent:

(i) A is Arens regular;

(ii) $A^{*}$ is a $\left(A^{* *}, \square\right)$-submodule of $A^{* * *}$;

(iii) $A^{*}$ is a $\left(A^{* *}, \diamond\right)$-submodule of $A^{* * *}$.

Let $\left(\pi_{1}, X, \pi_{2}\right)$ be a Banach $A$-module. As we mentioned just before Proposition 3.1, $\left(\pi_{2}^{* *}, X^{*}\right)$ and $\left(X^{*}, \pi_{1}^{r * * r}\right)$ are a left $\left(A^{* *}, \square\right)$-submodule and a right $\left(A^{* *}, \diamond\right)$-submodule of $X^{* * *}$, respectively. So if we assume that $A$ is Arens regular then $\left(\pi_{2}^{* *}, X^{*}\right)$ and $\left(X^{*}, \pi_{1}^{r * * r}\right)$ are left and right $A^{* *}$-modules, respectively. The next result deals with the question when $\left(\pi_{2}^{* *}, X^{*}, \pi_{1}^{r * * r}\right)$ is actually an $A^{* *}$-module.

Proposition 3.3. Let $A$ be Arens regular and let $\left(\pi_{1}, X, \pi_{2}\right)$ be a Banach $A$ module. Then $\left(\pi_{2}^{* *}, X^{*}, \pi_{1}^{r * * r}\right)$ is a Banach $A^{* *}$-module if and only if the bilinear map

$$
\theta_{x}: A \times A \longrightarrow X, \quad \theta_{x}(a, b)=\pi_{1}\left(a, \pi_{2}(x, b)\right)=\pi_{2}\left(\pi_{1}(a, x), b\right) \quad(a, b \in A),
$$

is regular, for all $x \in X$.

Proof. The triple $\left(\pi_{2}^{* *}, X^{*}, \pi_{1}^{r * * r}\right)$ is a Banach $A^{* *}$-module if and only if for all $a^{* *}, b^{* *} \in A^{* *}, x^{*} \in X^{*}$ and $x \in X$,

$$
\left\langle\pi_{1}{ }^{r * r}\left(\pi_{2}{ }^{* *}\left(b^{* *}, x^{*}\right), a^{* *}\right), x\right\rangle=\left\langle\pi_{2}{ }^{* *}\left(b^{* *}, \pi_{1}{ }^{r * * r}\left(x^{*}, a^{* *}\right)\right), x\right\rangle .
$$

Let $\left\{a_{\alpha}\right\}$ and $\left\{b_{\beta}\right\}$ be two nets in $A$ that converge to $a^{* *}$ and $b^{* *}$, respectively, in the $w^{*}$-topology of $A^{* *}$. Then a direct verification reveals that

$$
\begin{aligned}
\left\langle\pi_{1}{ }^{r * * r}\left(\pi_{2}{ }^{* *}\left(b^{* *}, x^{*}\right), a^{* *}\right), x\right\rangle & =\lim _{\alpha} \lim _{\beta}\left\langle x^{*}, \pi_{2}\left(\pi_{1}\left(a_{\alpha}, x\right), b_{\beta}\right)\right\rangle \\
& =\lim _{\alpha} \lim _{\beta}\left\langle x^{*}, \theta_{x}\left(a_{\alpha}, b_{\beta}\right)\right\rangle
\end{aligned}
$$

and

$$
\begin{aligned}
\left\langle\pi_{2}{ }^{* *}\left(b^{* *}, \pi_{1}^{r * * r}\left(x^{*}, a^{* *}\right)\right), x\right\rangle & =\lim _{\beta} \lim _{\alpha}\left\langle x^{*}, \pi_{1}\left(a_{\alpha}, \pi_{2}\left(x, b_{\beta}\right)\right)\right. \\
& =\lim _{\beta} \lim _{\alpha}\left\langle x^{*}, \theta_{x}\left(a_{\alpha}, b_{\beta}\right)\right\rangle .
\end{aligned}
$$

Thus $\left(\pi_{2}^{* *}, X^{*}, \pi_{1}^{r * * r}\right)$ is a Banach $A^{* *}$-module if and only if $\theta_{x}$ is regular, for all $x \in X$.

As a consequence, we give the following result of Bunce and Paschke, [6].

Corollary 3.4 [6, Proposition 1.1]. Let $A$ be a $C^{*}$-algebra and let $\left(\pi_{1}, X, \pi_{2}\right)$ be a Banach A-module. Then $\left(\pi_{2}^{* *}, X^{*}, \pi_{1}^{r * * r}\right)$ is a Banach $A^{* *}$-module. 
ProOf. By Proposition 3.3 it is enough to show that $\theta_{x}: A \times A \longrightarrow X$ is regular, for all $x \in X$. Applying Theorem 2.1, the regularity of $\theta_{x}$ is equivalent to the weak compactness of the linear mapping $a \mapsto \theta_{x}{ }^{*}\left(x^{*}, a\right): A \longrightarrow A^{*}$, which is guaranteed by the fact that every bounded linear map from a $C^{*}$-algebra to the predual of a $W^{*}$ algebra is automatically weakly compact (see [1, Corollary II 9]).

REMARKS 3.5. (i) If either $\pi_{1}$ or $\pi_{2}$ is regular then trivially $\theta_{x}$ is regular, for each $x \in X$. Therefore, in the Arens regular setting for $A$, using Proposition 3.3, $X^{*}$ is an $A^{* *}$-module, which is actually an $A^{* *}$-submodule of $X^{* * *}$ (see Proposition 3.1).

(ii) In the Arens regular setting for $A$, if $\theta_{x}$ is regular for each $x \in X$ then $\left(\pi_{2}^{* *}, X^{*}, \pi_{1}^{r * * r}\right)$ is a Banach $A^{* *}$-module by Proposition 3.3, which gives rise naturally the dual Banach $A^{* *}$-module $\left(\pi_{1}^{r * * * r}, X^{* *}, \pi_{2}^{* * *}\right)$. On the other hand we have the canonical Banach $A^{* *}$-modules $\left(\pi_{1}^{* * *}, X^{* *}, \pi_{2}^{* * *}\right)$ and $\left(\pi_{1}^{r * * * r}, X^{* *}, \pi_{2}^{r * * * r}\right)$. It is worth noting that the involved dual module does not coincide with the canonical modules, in general. However, it is obvious that it coincides with one of them if (and only if) either $\pi_{1}$ or $\pi_{2}$ is regular. It is shown in [6, Proposition 1.2] that the involved module actions coincide when $A$ is a $C^{*}$ algebra and $X^{*}$ is weakly sequentially complete; however, according to what we shall demonstrate in the following, under these conditions on $A$ and $X$ the module actions $\pi_{1}$ and $\pi_{2}$ are regular.

(iii) Let $\left(\pi_{1}, X, \pi_{2}\right)$ be a Banach $A$-module, if $A$ is a $C^{*}$-algebra and $X^{*}$ is weakly sequentially complete, then by [2, Theorem 4.2] the bounded linear mappings $a \longmapsto \pi_{1}^{*}\left(x^{*}, a\right)$ and $a \longmapsto \pi_{2}^{r *}\left(x^{*}, a\right)$ from $A$ to $X^{*}$ are weakly compact for all $x^{*} \in X^{*}$. Now applying Theorem 2.1 for $\pi_{1}$ and $\pi_{2}^{r}$ shows that $\pi_{1}$ and $\pi_{2}$ are regular. Note that the weak sequential completeness of $X^{*}$ is essential and cannot be removed in general. For instance, let $A$ be the $C^{*}$-algebra of compact operators on a separable, infinite-dimensional Hilbert space $H$ and let $X$ be the trace-class operators on $H$; then a direct verification reveals that the usual $A$-module action on $X$ is not regular (see the example just before in [6, Proposition 1.2]).

Dales et al. in [10, Proposition 4.5] (see also [4, Theorem 4] and [11, Theorem 3.1]) have shown that, if $A$ is Arens regular with a bounded left approximate identity, then $\pi^{r *}: A^{*} \times A \longrightarrow A^{*}$ is regular if and only if $A$ is reflexive as a Banach space. (Note that the equality $\pi^{* * * r * r}=\pi^{r * r * * *}$ which they used in [10, Proposition 4.5] is equivalent to the regularity of both $A$ and $\pi^{r *}$; see Corollary 2.2.) In the next proposition we generalize their result, which also shows that the hypothesis of Arens regularity of $A$ in [10, Proposition 4.5] is superfluous. Before proceeding, we recall that, when $\left(\pi_{1}, X\right)$ is a left Banach $A$-module, then a bounded net $\left\{e_{\alpha}\right\}$ in $A$ is said to be a left approximate identity for $X$, if $\pi_{1}\left(e_{\alpha}, x\right) \longrightarrow x$, for each $x \in X$. As a consequence of the so-called Cohen factorization theorem (see [8]), it is known that a bounded left approximate identity of $A$ is that of $X$ if and only if $\pi_{1}(A, X)=X$. The same situation occurs for the right Banach $A$-module $\left(X, \pi_{2}\right)$. 
Proposition 3.6. Let $\left(\pi_{1}, X\right)$ and $\left(X, \pi_{2}\right)$ be left and right Banach A-modules, respectively.

(i) If A has a bounded left approximate identity for $X$, then $\pi_{1}^{r *}$ is regular if and only if $X$ is reflexive.

(ii) If $A$ has a bounded right approximate identity for $X$, then $\pi_{2}^{*}$ is regular if and only if $X$ is reflexive.

PROOF. (i) If $X$ is reflexive, then

$$
\left(\pi_{1}^{r *}\right)^{* * * *}\left(A^{* *}, X^{* * *}\right)=\pi_{1}^{r * * * * *}\left(A^{* *}, X^{*}\right)=\pi_{1}^{r * *}\left(A^{* *}, X^{*}\right) \subseteq X^{*},
$$

now Theorem 2.1 implies that $\pi_{1}^{r *}$ is regular. For the converse, first note that, if $\left\{e_{\alpha}\right\}$ is a bounded left approximate identity for $X$ (in $A$ ), then a direct verification reveals that for every $x^{* *} \in X^{* *}, \pi_{1}^{r * *}\left(x^{* *}, e^{* *}\right)=x^{* *}$, in which $e^{* *}$ is a $w^{*}$-cluster point of $\left\{e_{\alpha}\right\}$ in $A^{* *}$. The latter identity in turn implies that $x^{* * *}=\pi_{1}^{r * * * *}\left(e^{* *}, x^{* * *}\right)$, for every $x^{* * *} \in X^{* * *}$. Now the regularity of $\pi_{1}^{r *}$ (again by Theorem 2.1) shows that $\pi_{1}^{r * * * *}\left(e^{* *}, x^{* * *}\right) \in X^{*}$. We thus have $x^{* * *} \in X^{*}$, or equivalently $X$ is reflexive. The proof of (ii) is very similar to that of (i).

\section{The second adjoint of a derivation}

Let $\left(\pi_{1}, X, \pi_{2}\right)$ be a Banach $A$-module. A bounded linear mapping $D: A \longrightarrow$ $X^{*}$ is said to be a derivation if $D(a b)=D(a) \cdot b+a \cdot D(b)$, for each $a, b \in A$, or equivalently

$$
D(a b)=\pi_{1}^{*}(D(a), b)+\pi_{2}^{r * r}(a, D(b)) .
$$

In this section we deal with the question of when the second adjoint $D^{* *}: A^{* *} \longrightarrow$ $X^{* * *}$ of $D: A \longrightarrow X^{*}$ is again a derivation. We recall from the beginning of the previous section that $\left(\pi_{2}^{* * * r * r}, X^{* * *}, \pi_{1}^{* * * *}\right)$ and $\left(\pi_{2}^{r * * * * r}, X^{* * *}, \pi_{1}^{r * * * r *}\right)$ are our canonical Banach $\left(A^{* *}, \square\right)$-module and $\left(A^{* *}, \diamond\right)$-module, respectively. Hence, $D^{* *}$ : $\left(A^{* *}, \square\right) \longrightarrow X^{* * *}$ is a derivation if and only if for every $a^{* *}, b^{* *} \in A^{* *}$,

$$
D^{* *}\left(a^{* *} \square b^{* *}\right)=\pi_{1}^{* * * *}\left(D^{* *}\left(a^{* *}\right), b^{* *}\right)+\pi_{2}^{* * * r * r}\left(a^{* *}, D^{* *}\left(b^{* *}\right)\right) .
$$

Similarly, $D^{* *}:\left(A^{* *}, \diamond\right) \longrightarrow X^{* * *}$ is a derivation if and only if

$$
D^{* *}\left(a^{* *} \diamond b^{* *}\right)=\pi_{1}^{r * * * r *}\left(D^{* *}\left(a^{* *}\right), b^{* *}\right)+\pi_{2}^{r * * * * r}\left(a^{* *}, D^{* *}\left(b^{* *}\right)\right) .
$$

We commence with the following lemma.

Lemma 4.1. Let $\left(\pi_{1}, X, \pi_{2}\right)$ be a Banach A-module, and let $D: A \longrightarrow X^{*}$ be a derivation. Then for all $a^{* *}, b^{* *} \in A^{* *}$,

(i) $D^{* *}\left(a^{* *} \square b^{* *}\right)=\pi_{1}^{* * * *}\left(D^{* *}\left(a^{* *}\right), b^{* *}\right)+\pi_{2}^{r * r * * *}\left(a^{* *}, D^{* *}\left(b^{* *}\right)\right)$ and

(ii) $D^{* *}\left(a^{* *} \diamond b^{* *}\right)=\pi_{1}^{* r * * * r}\left(D^{* *}\left(a^{* *}\right), b^{* *}\right)+\pi_{2}^{r * * * * r}\left(a^{* *}, D^{* *}\left(b^{* *}\right)\right)$. 
ProOf. (i) Let $\left\{a_{\alpha}\right\}$ and $\left\{b_{\beta}\right\}$ be two nets in $A$ that converge to $a^{* *}$ and $b^{* *}$ in the $w^{*}$-topology of $A^{* *}$, respectively. Then for each $x^{* *} \in X^{* *}$

$$
\begin{aligned}
\left\langle D^{* *}\left(a^{* *} \square b^{* *}\right), x^{* *}\right\rangle= & \lim _{\alpha} \lim _{\beta}\left\langle D\left(a_{\alpha} b_{\beta}\right), x^{* *}\right\rangle \\
= & \lim _{\alpha} \lim _{\beta}\left\langle\pi_{1}^{*}\left(D\left(a_{\alpha}\right), b_{\beta}\right)+\pi_{2}^{r * r}\left(a_{\alpha}, D\left(b_{\beta}\right)\right), x^{* *}\right\rangle \\
= & \lim _{\alpha} \lim _{\beta}\left\langle\pi_{1}^{* *}\left(x^{* *}, D\left(a_{\alpha}\right)\right), b_{\beta}\right\rangle \\
& +\lim _{\alpha} \lim _{\beta}\left\langle\pi_{2}^{r * r *}\left(x^{* *}, a_{\alpha}\right), D\left(b_{\beta}\right)\right\rangle \\
= & \lim _{\alpha}\left\langle\pi_{1}^{* *}\left(x^{* *}, D\left(a_{\alpha}\right)\right), b^{* *}\right\rangle+\lim _{\alpha}\left\langle\pi_{2}^{r * r *}\left(x^{* *}, a_{\alpha}\right), D^{* *}\left(b^{* *}\right)\right\rangle \\
= & \left\langle\pi_{1}^{* * *}\left(b^{* *}, x^{* *}\right), D^{* *}\left(a^{* *}\right)\right\rangle+\left\langle\pi_{2}^{r * r * *}\left(D^{* *}\left(b^{* *}\right), x^{* *}\right), a^{* *}\right\rangle \\
= & \left\langle\pi_{1}^{* * * *}\left(D^{* *}\left(a^{* *}\right), b^{* *}\right)+\pi_{2}^{r * r * * *}\left(a^{* *}, D^{* *}\left(b^{* *}\right)\right), x^{* *}\right\rangle .
\end{aligned}
$$

Hence $D^{* *}\left(a^{* *} \square b^{* *}\right)=\pi_{1}^{* * * *}\left(D^{* *}\left(a^{* *}\right), b^{* *}\right)+\pi_{2}^{r * r * * *}\left(a^{* *}, D^{* *}\left(b^{* *}\right)\right)$. A similar argument applies for (ii).

Dales et al. in the main theorem of their paper, [10, Theorem 7.1], have shown that the second adjoint $D^{* *}:\left(A^{* *}, \square\right) \longrightarrow A^{* * *}$ of the derivation $D$ : $A \longrightarrow A^{*}$ is a derivation if and only if $D^{* *}\left(A^{* *}\right) \cdot A^{* *} \subseteq A^{*}$, or equivalently $\pi^{r * * *}\left(D^{* *}\left(A^{* *}\right), A^{* *}\right) \subseteq A^{*}$, where $\pi$ is the multiplication of $A$. The next theorem extends this with a direct proof.

Theorem 4.2. Let $\left(\pi_{1}, X, \pi_{2}\right)$ be a Banach $A$-module and let $D: A \longrightarrow X^{*}$ be a derivation.

(i) $D^{* *}:\left(A^{* *}, \square\right) \longrightarrow X^{* * *}$ is a derivation if and only if $\pi_{2}^{* * * *}\left(D^{* *}\left(A^{* *}\right), X^{* *}\right)$ $\subseteq A^{*}$

(ii) $D^{* *}:\left(A^{* *}, \diamond\right) \longrightarrow X^{* * *}$ is a derivation if and only if $\pi_{1}^{r * * *}\left(D^{* *}\left(A^{* *}\right), X^{* *}\right)$ $\subseteq A^{*}$.

PROOF. We only prove (i). Let $a^{* *}, b^{* *} \in A^{* *}$ and $x^{* *} \in X^{* *}$ be arbitrary. Applying Lemma 4.1 and the equations just before it, one may deduce that $D^{* *}:\left(A^{* *}, \square\right) \longrightarrow$ $X^{* * *}$ is a derivation if and only if

$$
\left\langle\pi_{2}^{* * * r * r}\left(a^{* *}, D^{* *}\left(b^{* *}\right)\right), x^{* *}\right\rangle=\left\langle\pi_{2}^{r * r * * *}\left(a^{* *}, D^{* *}\left(b^{* *}\right)\right), x^{* *}\right\rangle,
$$

which holds if and only if

$$
\left\langle\pi_{2}^{* * * *}\left(D^{* *}\left(b^{* *}\right), x^{* *}\right), a^{* *}\right\rangle=\left\langle\pi_{2}^{r * r * *}\left(D^{* *}\left(b^{* *}\right), x^{* *}\right), a^{* *}\right\rangle,
$$

or equivalently,

$$
\pi_{2}^{* * * *}\left(D^{* *}\left(b^{* *}\right), x^{* *}\right)=\pi_{2}^{r * r * *}\left(D^{* *}\left(b^{* *}\right), x^{* *}\right) .
$$

It should be noted that $\pi_{2}^{* * * *}\left(D^{* *}\left(b^{* *}\right), x^{* *}\right) \in A^{* * *}$, while $\pi_{2}^{r * r * *}\left(D^{* *}\left(b^{* *}\right), x^{* *}\right)$ $\in A^{*}$ and also $\pi_{2}^{* * * *}\left(D^{* *}\left(b^{* *}\right), x^{* *}\right)_{\left.\right|_{A}}=\pi_{2}^{r * r * *}\left(D^{* *}\left(b^{* *}\right), x^{* *}\right)$. Thus $D^{* *}$ is a derivation if and only if $\pi_{2}^{* * * *}\left(D^{* *}\left(b^{* *}\right), x^{* *}\right) \in A^{*}$. 
As immediate consequences of the Theorem 4.2 we have the following corollaries.

Corollary 4.3. Let $\left(\pi_{1}, X, \pi_{2}\right)$ be a Banach $A$-module and let $D: A \longrightarrow X^{*}$ be a derivation. If $A$ is Arens regular then the following statements are equivalent:

(i) $D^{* *}: A^{* *} \longrightarrow X^{* * *}$ is a derivation;

(ii) $\pi_{2}^{* * * *}\left(D^{* *}\left(A^{* *}\right), X^{* *}\right) \subseteq A^{*}$;

(iii) $\pi_{1}^{r * * * *}\left(D^{* *}\left(A^{* *}\right), X^{* *}\right) \subseteq A^{*}$.

Corollary 4.4. Let $\left(\pi_{1}, X, \pi_{2}\right)$ be a Banach A-module, and let $D: A \longrightarrow X^{*}$ be a derivation.

(i) If both $\pi_{2}$ and $\pi_{2}^{r *}$ are Arens regular then $D^{* *}:\left(A^{* *}, \square\right) \longrightarrow X^{* * *}$ is a derivation.

(ii) If both $\pi_{1}$ and $\pi_{1}^{*}$ are Arens regular then $D^{* *}:\left(A^{* *}, \diamond\right) \longrightarrow X^{* * *}$ is a derivation.

PROOF. (i) If both $\pi_{2}$ and $\pi_{2}^{r *}$ are regular then $\pi_{2}^{* * * *}\left(X^{* * *}, X^{* *}\right) \subseteq A^{*}$ by Corollary 2.2. In particular, $\pi_{2}^{* * * *}\left(D^{* *}\left(A^{* *}\right), X^{* *}\right) \subseteq A^{*}$ and the conclusion follows from Theorem 4.2. A similar proof applies for (ii).

However, the regularity of both $\pi_{2}$ and $\pi_{2}^{r *}$ in (i) (and in turn $\pi_{1}$ and $\pi_{1}^{*}$ in (ii)) of the latter corollary ensures that $D^{* *}$ is a derivation, but it seems that these conditions impose rather a strong requirement on the module actions. As another application of Theorem 4.2, we give the following generalization of [10, Corollary 7.2(i)] which shares the imposed requirements on both module action and derivation.

Corollary 4.5. Let $\left(\pi_{1}, X, \pi_{2}\right)$ be a Banach A-module and let $D: A \longrightarrow X^{*}$ be a weakly compact derivation.

(i) If $\pi_{2}$ is regular then $D^{* *}:\left(A^{* *}, \square\right) \longrightarrow X^{* * *}$ is a derivation.

(ii) If $\pi_{1}$ is regular then $D^{* *}:\left(A^{* *}, \diamond\right) \longrightarrow X^{* * *}$ is a derivation.

Proof. (i) Since $D: A \longrightarrow X^{*}$ is weakly compact, $D^{* *}\left(A^{* *}\right) \subseteq X^{*}$. On the other hand the regularity of $\pi_{2}$ implies that $\pi_{2}^{* * *}\left(X^{*}, X^{* *}\right) \subseteq A^{*}$ (see Theorem 2.1). Therefore $\pi_{2}^{* * *}\left(D^{* *}\left(A^{* *}\right), X^{* *}\right) \subseteq A^{*}$, which means that $D^{* *}:\left(A^{* *}, \square\right) \longrightarrow X^{* * *}$ is a derivation.

We conclude this section with some observations on inner derivations. A linear mapping $D: A \longrightarrow X^{*}$ is said to be an inner derivation if $D(a)=x^{*} \cdot a-a \cdot x^{*}$ for some $x^{*} \in X^{*}$, or equivalently $D(a)=\pi_{1}^{*}\left(x^{*}, a\right)-\pi_{2}^{r * r}\left(a, x^{*}\right)$, for all $a \in A$. Then it follows trivially that for every $a^{* *} \in A^{* *}$,

$$
D^{* *}\left(a^{* *}\right)=\pi_{1}^{* * * *}\left(x^{*}, a^{* *}\right)-\pi_{2}^{r * * * *}\left(x^{*}, a^{* *}\right) .
$$

If, in addition, we assume that $\pi_{1}$ and $\pi_{2}$ are regular then Theorem 2.1 implies that the right-hand side of the latter equality belongs to $X^{*}$; thus $D^{* *}\left(A^{* *}\right) \subseteq X^{*}$ 
or, equivalently, $D$ is weakly compact. Furthermore, in the same situation the latter identity and the equalities $\pi_{1}^{* * * *}=\pi_{1}^{r * * * r *}$ and $\pi_{2}^{r * * * * r}=\pi_{2}^{* * * r * r}$ together imply that

$$
D^{* *}\left(a^{* *}\right)=\pi_{1}^{* * * *}\left(x^{*}, a^{* *}\right)-\pi_{2}^{* * * * r}\left(a^{* *}, x^{*}\right)
$$

and

$$
D^{* *}\left(a^{* *}\right)=\pi_{1}^{r * * * r *}\left(x^{*}, a^{* *}\right)-\pi_{2}^{r * * * * r}\left(a^{* *}, x^{*}\right),
$$

which means that both $D^{* *}:\left(A^{* *}, \square\right) \longrightarrow X^{* * *}$ and $D^{* *}:\left(A^{* *}, \diamond\right) \longrightarrow X^{* * *}$ are also inner derivations. We summarize these observations in the next result which is a generalization of [10, Proposition 6.1].

Proposition 4.6. Let $X$ be a A-module whose module actions are regular. Then every inner derivation $D: A \longrightarrow X^{*}$ is weakly compact; moreover, $D^{* *}$ : $\left(A^{* *}, \square\right) \longrightarrow X^{* * *}$ and $D^{* *}:\left(A^{* *}, \diamond\right) \longrightarrow X^{* * *}$ are also inner derivations.

\section{Acknowledgements}

This paper was completed while the second author was visiting the University of New South Wales in Australia. He would like to thank the School of Mathematics and Statistics, especially Professor Michael Cowling, for their generous hospitality. A valuable discussion with Professor Garth Dales during his short visit to Mashhad University is also acknowledged.

\section{References}

[1] C. A. Akemann, 'The dual space of an operator algebra', Trans. Amer. Math. Soc. 126 (1967), 286-302.

[2] C. A. Akemann, P. G. Dodds and J. L. B. Gamlen, 'Weak compactness in the dual space of a $C^{*}$-algebra', J. Funct. Anal. 10 (1972), 446-450.

[3] A. Arens, 'The adjoint of a bilinear operation', Proc. Amer. Math. Soc. 2 (1951), 839-848.

[4] N. Arikan, 'Arens regularity and reflexivity', Quart. J. Math. Oxford 32(2) (1981), 383-388.

[5] 'A simple condition ensuring the Arens regularity of bilinear mappings', Proc. Amer. Math. Soc. 84 (1982), 525-532.

[6] J. W. Bunce and W. L. Paschke, 'Derivations on a $C^{*}$-algebra and its double dual', J. Funct. Anal. 37 (1980), 235-247.

[7] P. Civin and B. Yood, 'The second conjugate space of a Banach algebra as an algebra', Pacific J. Math. 11 (1961), 847-870.

[8] H. G. Dales, Banach Algebras and Automatic Continuity, London Mathematical Society Monographs, 24 (Clarendon Press, Oxford, 2000).

[9] H. G. Dales and A. T.-M. Lau, 'The second duals of Beurling algebras', Mem. Amer. Math. Soc. 177(836) (2005).

[10] H. G. Dales, A. Rodríguez-Palacios and M. V. Velasco, 'The second transpose of a derivation', $J$. London Math. Soc. 64(2) (2001), 707-721.

[11] A. Ulger, 'Weakly compact bilinear forms and Arens regularity', Proc. Amer. Math. Soc. 101 (1987), 697-704.

[12] N. J. Young, 'The irregularity of multiplication in group algebras', Quart. J. Math. Oxford 24(2) (1973), 59-62. 
S. MOHAMMADZADEH, Department of Mathematics, Ferdowsi University of Mashhad, P.O. Box 91775-1159, Mashhad, Iran

e-mail: somohammadzad@yahoo.com

H. R. E. VISHKI, Department of Mathematics, Ferdowsi University of Mashhad, P.O. Box 91775-1159, Mashhad, Iran

and

Centre of Excellence in Analysis on Algebraic Strutures (CEAAS),

Ferdowski University of Mashhad, Mashhad, Iran

e-mail:vishki@ferdowsi.um.ac.ir 